\title{
Predictive Model of Type 2 Diabetes Remission after Metabolic Surgery in Chinese Patients
}

\author{
Yufang Luo, Zi Guo $(\mathbb{D}$, Honghui He, Youbo Yang, Shaoli Zhao, and Zhaohui Mo \\ Department of Endocrinology, Third Xiangya Hospital of Central South University \& Diabetic Foot Research Center of Central \\ South University, Changsha, Hunan Province 410013, China
}

Correspondence should be addressed to Zhaohui Mo; easd04mzh@gmail.com

Received 16 June 2020; Revised 27 August 2020; Accepted 18 September 2020; Published 31 October 2020

Academic Editor: Tatsuya Kin

Copyright (c) 2020 Yufang Luo et al. This is an open access article distributed under the Creative Commons Attribution License, which permits unrestricted use, distribution, and reproduction in any medium, provided the original work is properly cited.

\begin{abstract}
Introduction. Metabolic surgery is an effective treatment for type 2 diabetes (T2D). At present, there is no authoritative standard for predicting postoperative T2D remission in clinical use. In general, East Asian patients with T2D have a lower body mass index and worse islet function than westerners. We aimed to look for clinical predictors of T2D remission after metabolic surgery in Chinese patients, which may provide insights for patient selection. Methods. Patients with T2D who underwent metabolic surgery at the Third Xiangya Hospital between October 2008 and March 2017 were enrolled. T2D remission was defined as an HbA1c level below $6.5 \%$ and an FPG concentration below $7.1 \mathrm{mmol} / \mathrm{L}$ for at least one year in the absence of antidiabetic medications. Results. (1) Independent predictors of short-term T2D remission (1-2 years) were age and C-peptide area under the curve (C-peptide AUC); independent predictors of long-term T2D remission (4-6 years) were C-peptide AUC and fasting plasma glucose (FPG). (2) The optimal cutoff value for C-peptide AUC in predicting T2D remission was $30.93 \mathrm{ng} / \mathrm{ml}$, with a specificity of $67.3 \%$ and sensitivity of $75.8 \%$ in the short term and with a specificity of $61.9 \%$ and sensitivity of $81.5 \%$ in the long term, respectively. The areas under the ROC curves are 0.674 and 0.623 in the short term and long term, respectively. (3) We used three variables (age, C-peptide AUC, and FPG) to construct a remission prediction score (ACF), a multidimensional 9-point scale, along which greater scores indicate a better chance of T2D remission. We compared our scoring system with other reported models (ABCD, DiaRem, and IMS). The ACF scoring system had the best distribution of patients and prognostic significance according to the ROC curves. Conclusion. Presurgery age, C-peptide AUC, and FPG are independent predictors of T2D remission after metabolic surgery. Among these, C-peptide AUC plays a decisive role in both short- and long-term remission prediction, and the optimal cutoff value for C-peptide AUC in predicting T2D remission was $30.93 \mathrm{ng} / \mathrm{ml}$, with moderate predictive values. The ACF score is a simple reliable system that can predict T2D remission among Chinese patients.
\end{abstract}

\section{Introduction}

The number of patients with type 2 diabetes (T2D) and obesity is increasing worldwide. According to the latest epidemiology reports, the prevalence of T2D in China has risen to $10.4 \%$ [1]. Furthermore, it is predicted that by 2025 , more than $60 \%$ of patients with T2D worldwide will be in Asian countries [2]. Currently, usual therapy for most patients with T2D and obesity is lifestyle and pharmacological interventions. In recent decades, metabolic surgery (MS) has been widely accepted as a method of treatment for T2D. Several clinical randomized controlled trials indicated that, in comparison to conventional therapy, MS is not limited to improving obesity and T2D and effectively alleviates comorbidities such as hypertension and dyslipidemia, thus reducing cardiovascular-related mortality [3-9]. The mechanisms underlying MS for the treatment of T2D include food restriction, promotion of incretin secretion, changes in bile acid composition, and changes in intestinal microbiota [10-13]. The most common surgical procedures are gastrointestinal bypass (GB) and sleeve gastrectomy (SG) [14]. The development of perioperative care and laparoscopic techniques guarantees the safety of metabolic 
surgery, which has been adopted by T2D prevention guidelines in numerous countries.

As MS remains the most effective treatment for obesity and T2D, several studies have investigated the factors affecting T2D outcomes after surgery. Studies have demonstrated that race, age, duration of T2D, weight, body mass index (BMI), glycosylated hemoglobin (HbAlc) level, fasting plasma glucose (FPG) level, fasting C-peptide level (FCP) concentration, preoperative insulin use, and hypertension may affect the outcomes of MS [15-23]. Recently, scholars attempted to construct models $[24,25]$ and scoring systems to predict the resolution of T2D after bariatric surgery, such as the ABCD (age, BMI, C-peptide, and duration of T2D) [26], Integrated Medical Services (duration of T2D, preoperative number of diabetes medications, insulin use, and HbA1c) [27], DiaRem (age, HbA1c, antidiabetic drugs, and insulin treatment) [28], and Diabetes Risk Score (age, duration of T2D, BMI, microvascular and macrovascular complications, insulin use, and stimulated C-peptide level) [29], to predict the resolution of T2D after bariatric surgery. Variations in these reports may be due to differences in designs, populations, follow-up times, and operation procedures, and no authoritative standard for prediction is yet available for clinical use.

Previous reports have shown that East Asian patients with newly diagnosed T2D have lower BMIs and worse $\beta$-cell function than Western patients [30]. According to recently released guidelines, the eligibility for bariatric surgery in Asian patients with T2D, which is based on BMI, should be lowered to $27.5 \mathrm{~kg} / \mathrm{m}^{2}$ [31,32]. However, the islet function index has not been included in the selection of MS indications. Although numerous studies have emphasized the value of $\beta$-cell function in T2D remission prediction $[22,23,26,33]$, only a limited number of studies have focused on comprehensive indexes of islet function [34]. To providing a better understanding of $\beta$-cell function in the remission of Chinese patients with T2D, we performed a study applying various indicators that reflect islet function. Our study aimed to explore the predictive factors for T2D remission in the short term and long term after MS and to further establish a remission prediction score system based on mainland Chinese patients. A better understanding of the predictors for MS will help guide the choice of T2D therapy.

\section{Materials and Methods}

2.1. Patient Population. This prospective cohort study included 87 patients with T2D undergoing MS as inpatients in the Department of Endocrinology between October 2008 and March 2017. T2D was defined according to the 1999 World Health Organization (WHO) diagnostic criteria for $\mathrm{T} 2 \mathrm{D}$ or the preoperative use of diabetic medication. The inclusion criteria were as follows: (1) age between 18 and 70 years; (2) before May 2011, BMI $\geq 24 \mathrm{~kg} / \mathrm{m}^{2}$ (before the promulgation of the Chinese guidelines for $\mathrm{T} 2 \mathrm{D}$ ), and $\mathrm{BMI}$ $\geq 27.5 \mathrm{~kg} / \mathrm{m}^{2}$ thereafter; and (3) clinical diagnosis of T2D for no more than 15 years. The exclusion criteria were as follows: (1) abuse of alcohol or drugs or uncontrollable mental illness; (2) severe organ dysfunction or other diseases related to surgery intolerance; (3) combined acute complications such as diabetic ketoacidosis and a hyperosmolar hyperglycemic state; and (4) patients with type 1 diabetes, gestational diabetes, or other types of diabetes. The study was approved by the Third Xiangya Hospital Ethics Committee.

2.2. Data Collection. Data were obtained from the outpatient and inpatient departments. Age, duration of T2D, blood pressure, glucose, C-peptide, insulin, HbAlc, urinary microalbumin (UmAlb), and lipid biomarkers were documented before surgery and at the follow-up visits. Loss to follow-up was defined by no current available contact information or no response to three-time inquiries by phone. The main reasons for failing to participate in the follow-ups included an incorrect phone number, unaffordable medical expenses, migration, and long distance.

2.3. Indexes of Islet Function. This study included multiple indexes of $\beta$-cell function [35], including fasting C-peptide (FCP), C-peptide area under the curve (C-peptide AUC), first- and second-phase insulin secretion indexes $(\Delta \mathrm{C} 30$ and $\Delta \mathrm{C} 120)$, insulinogenic indexes $(\Delta \mathrm{C} 30 / \Delta \mathrm{G} 30$ and $\Delta \mathrm{C} 120 /$ $\Delta$ G120), homeostasis model assessment of $\beta$-cell function (HOMA- $\beta$ ), and homeostasis model assessment of insulin resistance (HOMA-IR). To eliminate the influence of exogenous insulin use, we used C-peptide as a substitute for insulin. The calculation of HOMA- $\beta$ and HOMA-IR was proposed in a previous paper [36]. The formulas were as defined as follows:

$$
\begin{aligned}
\Delta C 30 & =\mathrm{C}-\text { peptide }(30 \mathrm{~min})-\mathrm{FCP}, \\
\Delta C 120 & =\mathrm{C}-\text { peptide }(120 \mathrm{~min})-\mathrm{FCP}, \\
\frac{\Delta C 30}{\Delta G 30} & =\frac{\Delta C 30}{[\text { glucose }(30 \mathrm{~min})-\mathrm{FPG}]}, \\
\frac{\Delta C 120}{\Delta G 120}= & \frac{\Delta C 120}{[\text { glucose }(120 \mathrm{~min})-\mathrm{FPG}]}, \\
\mathrm{HOMA}-\beta(\mathrm{CP}, \mathrm{DM})= & 0.27 \times \frac{\mathrm{FCP}}{(\mathrm{FPG}-3.5)} \%, \\
\mathrm{HOMA}-\mathrm{IR}= & \frac{1.5+\mathrm{FCP} \times \mathrm{FPG}}{2800}, \\
\mathrm{C}-\text { peptide } A U C= & 0.25 \times \mathrm{FCP}+4 \times \mathrm{C} \\
& - \text { peptide }(30 \mathrm{~min})+3 \times \mathrm{C} \\
& - \text { peptide }(120 \mathrm{~min}) .
\end{aligned}
$$

2.4. Outcome Evaluation. T2D remission was defined as an HbA1c level below 6.5\% and an FPG concentration below $7.1 \mathrm{mmol} / \mathrm{L}$ for at least one year in the absence of antidiabetic medications. Recurrence was defined as the return of FPG or 
HbAlc levels to the diabetic range $(\geq 7.0 \mathrm{mmol} / \mathrm{L}$ and $\geq 6.5 \%$, respectively) or the need for antidiabetic medication after initial remission. Short-term remission was defined as T2D remission in the first 1-2 years after surgery, and long-term remission was defined as T2D remission for 4-6 years after surgery.

\subsection{Scoring System}

2.5.1. ABCD Score. Four parameters, including age, BMI, C-peptide level, and duration of T2D, were used for scoring (total points ranged from 0 to 10) [26]. One point was used for age. Each of the remaining three variables had 4 categories, and $0-3$ points were assigned. Patients with higher $\mathrm{ABCD}$ scores were predicted to have a higher probability of T2D remission after surgery.

2.5.2. DiaRem Score. Four parameters, including age, $\mathrm{HbA1c}$, anti-T2D drugs, and insulin, were used for scoring (total points ranged from 0 to 22) [28]. The age score ranged from 0 to 3, and $\mathrm{HbAlc}$ ranged from 0 to 6 due to different categories. A 3-point score was used for using anti-T2D drug use, and a 10-point score was used for insulin use. Patients with lower DiaRem scores were predicted to have a higher probability of $\mathrm{T} 2 \mathrm{D}$ remission after surgery.

2.5.3. Individualized Metabolic Surgery (IMS) Score. Four parameters, including number of T2D medications, insulin use, T2D duration, and $\mathrm{HbA1c}$, were used for scoring [27]. A constructed nomogram allowed for the classification of diabetes severity into mild, moderate, and severe groups. Patients with lower IMS scores were predicted to have a higher probability of T2D remission after surgery.

2.6. Statistical Analysis. All analyses were performed using IBM SPSS for Windows, version 23.0 (IBM Corp., Armonk, $\mathrm{NY}$ ). Continuous variables were expressed as the means with $\mathrm{SD}$ or with median. Baseline comparisons were performed using chi-square tests and $t$-tests. Relationships between two continuous variables were tested by Pearson correlation analysis. Univariate regression analysis was used to screen for possible predictors, and binary regression analysis was used to identify the independent predictors of T2D remission. Receiver operating characteristic (ROC) curve analysis was performed to determine the diagnostic value, and the optimal cutoff was specified with Youden's J index (=sensitivity + specificity -1$)$. All statistical tests were tested two sided, and $P<0.05$ was considered statistically significant.

\section{Results}

3.1. Baseline Characteristics. This study included a total of 87 patients with T2D (60 males and 27 females). The average age of the patients was $44.2 \pm 11.2$ years, and the T2D duration was $6.4 \pm 4.8$ years. The average patients' BMI was $31.29 \pm 6.51 \mathrm{~kg} / \mathrm{m}^{2}$. There were 9 patients $(10.3 \%)$ taking lifestyle interventions without medicine before surgery, 32 patients $(36.8 \%)$ using oral antidiabetic drugs, 20 patients (23.0\%) using insulin, and 26 patients (29.9\%) using oral antidiabetic drugs combined with insulin, respectively. GB was performed in 62 patients, while the others received SG. The patients' baseline data are shown in Table 1. Patients who underwent SG were younger and heavier and had better residual $\beta$-cell function than the patients who underwent GB $(P<0.05)$.

Here, we analysed the relationship between the course of disease and $\beta$-cell function indexes. Duration of T2D was negatively correlated with FCP $(r=-0.257, P=0.016), \Delta \mathrm{C} 30 /$ $\triangle \mathrm{G} 30(r=-0.279, P=0.011)$, HOMA-IR $(\mathrm{CP})(r=-0.270$, $P=0.012)$, and C-peptide AUC $(r=-0.257, P=0.020)$. No correlation was observed between duration of T2D and $\triangle \mathrm{C} 30$ $(r=-0.149, \quad P=0.181), \quad \Delta \mathrm{C} 120 \quad(r=-0.138, \quad P=0.202)$, $\Delta \mathrm{C} 120 / \Delta \mathrm{G} 120(r=-0.067, P=0.535)$, and HOMA $-\beta$ (CP, DM) $(r=-0.071, P=0.515)$.

3.2. Predictors of Short-Term T2D Remission. A total of 53 of the $87(60.92 \%)$ patients achieved T2D remission at $1-2$ years of follow-up. Among them, there were 23 patients who underwent SG and 30 patients who underwent GB, respectively. Univariate regression showed that patients with a younger age, higher BMI, shorter duration of T2D, SG, no presurgical insulin use, higher levels of FCP, second-phase insulin secretion indexes $(\triangle \mathrm{C} 120)$, HOMA- $\beta$ (CP, DM), HOMA-IR (CP), and C-peptide AUC values (the values of $\beta$-cell function indexes were grouped by median) had higher $\mathrm{T} 2 \mathrm{D}$ remission rates than other patients 1-2 years after surgery (Table 2). There was no significant difference in remission rates among groups classified by sex, lipid profiles, umAlb, HbA1c, FPG, first-phase insulin secretion indexes $(\Delta \mathrm{C} 30)$, or insulinogenic indexes $(\Delta \mathrm{C} 30 / \Delta \mathrm{G} 30$ and $\Delta \mathrm{C} 120 /$ $\Delta \mathrm{G} 120)$. Considering that the difference in baseline characteristics between the two surgical procedure groups was significant, we excluded surgical procedures when performing the binary logistic regression. Taking age, duration, BMI, FPG, and C-peptide AUC into the binary regression, the results showed that C-peptide AUC and age were independent predictors of short-term T2D remission after MS (Table 3).

3.3. Predictors of Prolonged T2D Remission. Among 72 patients who underwent surgery for more than 4 years, 50 of them (69.44\%) underwent long-term follow-up after the surgery. There were $19(38.00 \%)$ patients who remained in prolonged remission; however, 5 patients who achieved remission at 1-2 years after surgery experienced T2D recurrence in the long term. Univariate analysis showed that FPG, HbA1c, and C-peptide AUC values were factors affecting T2D remission 4-6 years after surgery, while there was no significant difference in age, sex, duration, surgical procedures, BMI, lipid profiles, FCP, $\Delta$ C30, $\Delta$ C120, $\Delta$ C30/ $\Delta \mathrm{G} 30, \Delta \mathrm{C} 120 / \Delta \mathrm{G} 120$, HOMA- $\beta$ (CP, DM), HOMA-IR (CP), presurgery insulin use, and UmAlb (Table 4). Binary regression analysis showed that level of C-peptide AUC and 
TABLE 1: Characteristics of the patients at baseline.

\begin{tabular}{|c|c|c|c|c|c|c|c|}
\hline \multirow{2}{*}{ Parameters } & \multicolumn{2}{|r|}{ Overall $(N=87)$} & \multicolumn{2}{|r|}{$\mathrm{GB}(N=62)$} & \multicolumn{2}{|r|}{$\mathrm{SG}(N=25)$} & \multirow{2}{*}{$P$ value } \\
\hline & $N$ & Mean \pm SD (median) & $N$ & Mean \pm SD (median) & $N$ & Mean \pm SD (median) & \\
\hline Age (yr) & 87 & $44.2 \pm 11.2(45)$ & 62 & $46.8 \pm 9.6(47)$ & 25 & $37.7 \pm 12.5$ & $<0.001^{*}$ \\
\hline Duration (yr) & 87 & $6.4 \pm 4.8(6)$ & 62 & $6.7 \pm 4.7(6.5)$ & 25 & $5.7 \pm 5.0$ & 0.286 \\
\hline BMI $\left(\mathrm{kg} / \mathrm{m}^{2}\right)$ & 87 & $31.29 \pm 6.51(29.41)$ & 62 & $28.96 \pm 4.84(28.04)$ & 25 & $37.09 \pm 6.57(35.64)$ & $<0.001^{*}$ \\
\hline SBP (mmHg) & 87 & $136.1 \pm 16.5(135)$ & 62 & $135.9 \pm 16.8(135)$ & 25 & $136.5 \pm 15.9(135)$ & 0.884 \\
\hline $\mathrm{DBP}(\mathrm{mmHg})$ & 87 & $86.1 \pm 11.7(81)$ & 62 & $85.6 \pm 10.7(80)$ & 25 & $87.4 \pm 14.0(83)$ & 0.543 \\
\hline TG $(\mathrm{mmol} / \mathrm{L})$ & 87 & $2.63 \pm 2.40(1.93)$ & 62 & $2.67 \pm 2.50(1.91)$ & 25 & $2.53 \pm 2.19(1.93)$ & 0.870 \\
\hline CHOL (mmol/L) & 87 & $4.85 \pm 1.08(4.87)$ & 62 & $4.87 \pm 1.12(4.87)$ & 25 & $4.79 \pm 1.06(4.89)$ & 0.974 \\
\hline $\mathrm{HDL}(\mathrm{mmol} / \mathrm{L})$ & 87 & $1.11 \pm 0.26(1.09)$ & 62 & $1.14 \pm 0.27(1.13)$ & 25 & $1.03 \pm 0.24(1.01)$ & $0.030^{*}$ \\
\hline $\mathrm{LDL}(\mathrm{mmol} / \mathrm{L})$ & 87 & $2.66 \pm 0.84(2.64)$ & 62 & $2.67 \pm 0.82(2.67)$ & 25 & $2.62 \pm 0.91(2.62)$ & 0.955 \\
\hline FPG (mmol/L) & 87 & $7.61 \pm 2.22(7.26)$ & 62 & $7.57 \pm 1.97(7.39)$ & 25 & $7.73 \pm 2.78(7.17)$ & 0.760 \\
\hline HbAlc (\%) & 87 & $8.01 \pm 1.70(7.9)$ & 62 & $8.18 \pm 1.64(7.9)$ & 25 & $7.58 \pm 1.80(7.8)$ & 0.108 \\
\hline FCP (ng/mL) & 87 & $2.68 \pm 1.84(2.63)$ & 62 & $2.25 \pm 1.88(1.66)$ & 25 & $3.73 \pm 1.22(3.38)$ & $<0.001^{*}$ \\
\hline$\Delta \mathrm{C} 30(\mathrm{ng} / \mathrm{mL})$ & 82 & $1.58 \pm 1.70(1.01)$ & 59 & $1.24 \pm 1.53(0.75)$ & 23 & $2.44 \pm 1.85(1.62)$ & $0.001^{*}$ \\
\hline$\Delta \mathrm{C} 120(\mathrm{ng} / \mathrm{mL})$ & 87 & $4.57 \pm 6.80(3.08)$ & 62 & $3.98 \pm 7.74(2.51)$ & 25 & $6.04 \pm 3.25(5.38)$ & $<0.001^{*}$ \\
\hline$\Delta \mathrm{C} 30 / \Delta \mathrm{G} 30(\mu \mathrm{g} / \mathrm{mmol})$ & 82 & $0.36 \pm 0.46(0.27)$ & 59 & $0.28 \pm 0.39(0.15)$ & 23 & $0.58 \pm 0.55(0.44)$ & $0.003^{*}$ \\
\hline$\Delta \mathrm{C} 120 / \Delta \mathrm{G} 120(\mu \mathrm{g} / \mathrm{mmol})$ & 87 & $0.71 \pm 1.35(0.42)$ & 62 & $0.53 \pm 1.07(0.31)$ & 25 & $1.16 \pm 1.84(0.73)$ & $<0.001^{*}$ \\
\hline HOMA- $\beta(\mathrm{CP}, \mathrm{DM})(\%)$ & 87 & $72.48 \pm 59.97(52.71)$ & 62 & $57.80 \pm 48.95(41.14)$ & 25 & $108.87 \pm 69.71(94.97)$ & $<0.001^{*}$ \\
\hline HOMA-IR (CP) & 87 & $2.49 \pm 1.93(2.08)$ & 62 & $2.14 \pm 1.98(1.43)$ & 25 & $3.35 \pm 1.55(3.06)$ & $<0.001^{*}$ \\
\hline C-peptide AUC (ng/ml) & 82 & $38.90 \pm 38.89(30.93)$ & 59 & $33.22 \pm 36.52(24.95)$ & 23 & $53.47 \pm 20.06(52.77)$ & $<0.001^{*}$ \\
\hline UmAlb (mg/L) & 74 & $89.85 \pm 142.95(31.95)$ & 56 & $71.32 \pm 97.20(31.50)$ & 18 & $147.49 \pm 229.20(34.50)$ & 0.545 \\
\hline
\end{tabular}

Data are presented as the mean \pm standard deviation (median). SBP: systolic blood pressure; DBP: diastolic blood pressure; TG: triglycerides; CHOL: cholesterol; HDL: high-density lipoprotein; LDL: low-density lipoprotein; FPG: fasting plasma glucose; FCP: fasting C-peptide; C-peptide AUC: C-peptide area under the curve; UmAlb: urinary microalbumin. ${ }^{*} P<0.05$.

TABLE 2: Univariate analysis of remission predictors 1-2 years after surgery.

\begin{tabular}{|c|c|c|c|}
\hline & Remission group $(N=53)$ & Nonremission group $(N=34)$ & $P$ value \\
\hline Age (yr) & & & $<0.001^{*}$ \\
\hline$<40$ & $22(91.67 \%)$ & $2(8.33 \%)$ & \\
\hline$\geq 40$ & $31(49.21 \%)$ & $32(50.79 \%)$ & \\
\hline Gender & & & 0.101 \\
\hline Female & $13(48.15 \%)$ & $14(51.85 \%)$ & \\
\hline Male & $40(66.67 \%)$ & $20(33.33 \%)$ & \\
\hline Duration (yr) & & & $0.011^{*}$ \\
\hline$\leq 5$ & $32(74.42 \%)$ & $11(25.58 \%)$ & \\
\hline$>5$ & $21(47.73 \%)$ & $23(52.27 \%)$ & \\
\hline Surgical procedures & & & $<0.001^{*}$ \\
\hline Sleeve gastrectomy & $23(92.00 \%)$ & $2(8.00 \%)$ & \\
\hline Gastric bypass & $30(48.39 \%)$ & $32(51.62 \%)$ & \\
\hline BMI $\left(\mathrm{kg} / \mathrm{m}^{2}\right)$ & & & $<0.001^{*}$ \\
\hline$<27.5$ & $12(41.38 \%)$ & $17(58.62 \%)$ & \\
\hline $27.5-32.5$ & $20(55.56 \%)$ & $16(44.44 \%)$ & \\
\hline$\geq 32.5$ & $21(95.45 \%)$ & $1(4.55 \%)$ & \\
\hline $\mathrm{TG}(\mathrm{mmol} / \mathrm{L})$ & & & 0.493 \\
\hline$\geq 1.7$ & $32(58.18 \%)$ & $23(41.82 \%)$ & \\
\hline$<1.7$ & $21(65.63 \%)$ & $11(34.37 \%)$ & \\
\hline $\mathrm{CHOL}(\mathrm{mmol} / \mathrm{L})$ & & & 0.426 \\
\hline$\geq 4.14$ & $40(63.49 \%)$ & $23(36.51 \%)$ & \\
\hline$<4.14$ & $13(54.17 \%)$ & $11(45.83 \%)$ & \\
\hline $\mathrm{LDL}(\mathrm{mmol} / \mathrm{L})$ & & & 0.534 \\
\hline$\geq 2.59$ & $26(57.78 \%)$ & $19(42.22 \%)$ & \\
\hline$<2.59$ & $27(64.29 \%)$ & $15(35.71 \%)$ & \\
\hline $\mathrm{HDL}(\mathrm{mmol} / \mathrm{L})$ & & & 0.279 \\
\hline$>1.29$ & $11(73.33 \%)$ & $4(26.67 \%)$ & \\
\hline$\leq 1.29$ & $42(58.33 \%)$ & $30(41.67 \%)$ & \\
\hline $\mathrm{FPG}(\mathrm{mmol} / \mathrm{L})$ & & & 0.099 \\
\hline$<7.0$ & $25(71.43 \%)$ & $10(28.57 \%)$ & \\
\hline$\geq 7.0$ & $28(53.85 \%)$ & $24(46.15 \%)$ & \\
\hline
\end{tabular}


TABle 2: Continued.

\begin{tabular}{|c|c|c|c|}
\hline & Remission group $(N=53)$ & Nonremission group $(N=34)$ & $P$ value \\
\hline HbAlc (\%) & & & 0.270 \\
\hline$<6.5$ & $13(76.47 \%)$ & $4(25.53 \%)$ & \\
\hline $6.5 \leq \mathrm{HbA} 1 \mathrm{c}<9$ & $27(60.00 \%)$ & $18(40.00 \%)$ & \\
\hline$\geq 9$ & $13(52.00 \%)$ & $12(48.00 \%)$ & \\
\hline $\mathrm{FCP}(\mathrm{ng} / \mathrm{mL})$ & $(N=53)$ & $(N=34)$ & $0.011^{*}$ \\
\hline$\leq 2.63$ & $21(47.73 \%)$ & $23(52.27 \%)$ & \\
\hline$>2.63$ & $32(74.42 \%)$ & $11(25.58 \%)$ & \\
\hline$\Delta \mathrm{C} 30(\mathrm{ng} / \mathrm{mL})$ & $(N=49)$ & $(N=33)$ & 0.499 \\
\hline$<1.01$ & $23(56.10 \%)$ & $18(43.90 \%)$ & \\
\hline$\geq 1.01$ & $26(63.41 \%)$ & $15(36.59 \%)$ & \\
\hline$\Delta \mathrm{C} 120(\mathrm{ng} / \mathrm{mL})$ & $(N=53)$ & $(N=34)$ & $0.035^{*}$ \\
\hline$\leq 3.08$ & $22(50.00 \%)$ & $22(50.00 \%)$ & \\
\hline$>3.08$ & $31(72.09 \%)$ & $12(27.91 \%)$ & \\
\hline$\Delta \mathrm{C} 30 / \Delta \mathrm{G} 30(\mu \mathrm{g} / \mathrm{mmol})$ & $(N=49)$ & $(N=33)$ & 0.499 \\
\hline$\leq 0.27$ & $23(56.10 \%)$ & $18(43.90 \%)$ & \\
\hline$>0.27$ & $26(63.41 \%)$ & $15(36.59 \%)$ & \\
\hline$\Delta \mathrm{C} 120 / \Delta \mathrm{G} 120(\mu \mathrm{g} / \mathrm{mmol})$ & $(N=53)$ & $(N=34)$ & 0.095 \\
\hline$\leq 0.42$ & $23(52.27 \%)$ & $21(47.73 \%)$ & \\
\hline$>0.42$ & $30(69.77 \%)$ & $13(30.22 \%)$ & \\
\hline HOMA- $\beta(\mathrm{CP}, \mathrm{DM})(\%)$ & $(N=53)$ & $(N=34)$ & $0.003^{*}$ \\
\hline$\leq 52.71$ & $20(45.45 \%)$ & $24(54.55 \%)$ & \\
\hline$>52.71$ & $33(76.74 \%)$ & $10(23.26 \%)$ & \\
\hline HOMA-IR (CP) & $(N=53)$ & $(N=34)$ & $0.011^{*}$ \\
\hline$\leq 2.08$ & $21(47.73 \%)$ & $23(52.27 \%)$ & \\
\hline$>2.08$ & $32(74.42 \%)$ & $11(25.58 \%)$ & \\
\hline C-peptide AUC (ng/ml) & $(N=49)$ & $(N=33)$ & $<0.001^{*}$ \\
\hline$\leq 30.93$ & $16(39.02 \%)$ & $25(60.98 \%)$ & \\
\hline$>30.93$ & $33(80.49 \%)$ & $8(19.51 \%)$ & \\
\hline $\operatorname{UmAlb}(\mathrm{mg} / \mathrm{L})$ & $(N=42)$ & $(N=32)$ & 0.687 \\
\hline$<20$ & $15(60.00 \%)$ & $10(40.00 \%)$ & \\
\hline$\geq 20$ & $27(55.10 \%)$ & $22(44.90 \%)$ & \\
\hline Preoperative insulin use & & & $<0.001^{*}$ \\
\hline Yes & $20(43.48 \%)$ & $26(56.52 \%)$ & \\
\hline No & $33(80.49 \%)$ & $8(19.52 \%)$ & \\
\hline
\end{tabular}

${ }^{*} P<0.05$; indexes of islet function ((FCP, $\Delta \mathrm{C} 30, \Delta \mathrm{C} 120, \Delta \mathrm{C} 30 / \Delta \mathrm{G} 30, \Delta \mathrm{C} 120 / \Delta \mathrm{G} 120$, HOMA- $\beta$ (CP, DM), HOMA-IR (CP), and C-peptide AUC) were grouped by median.

TABLE 3: Binary logistic regression of short-term remission predictors.

\begin{tabular}{|c|c|c|c|}
\hline & $P$ value & OR & $\mathrm{CI}$ \\
\hline $\begin{array}{l}\text { C-peptide AUC (ng/ml) } \\
\quad \leq 30.93 \\
>30.93\end{array}$ & $\begin{array}{l}0.002^{*} \\
\text { Reference }\end{array}$ & 4.530 & $1.581-12.983$ \\
\hline $\begin{array}{l}\text { Age }(\mathrm{yr}) \\
\quad \geq 40 \\
<40\end{array}$ & $\begin{array}{l}0.012^{*} \\
\text { Reference }\end{array}$ & 7.705 & $1.567-37.874$ \\
\hline $\begin{array}{l}\text { BMI }\left(\mathrm{kg} / \mathrm{m}^{2}\right) \\
\quad<27.5 \\
27.5-32.5 \\
\geq 27.5\end{array}$ & $\begin{array}{c}0.136 \\
\text { Reference }\end{array}$ & & \\
\hline $\begin{array}{l}\text { Duration (yr) } \\
\quad \leq 5 \\
\quad>5\end{array}$ & $\begin{array}{c}0.097 \\
\text { Reference }\end{array}$ & & \\
\hline $\begin{array}{l}\mathrm{FPG}(\mathrm{mmol} / \mathrm{L}) \\
\quad<7.0 \\
\quad \geq 7.0\end{array}$ & $\begin{array}{c}0.279 \\
\text { Reference }\end{array}$ & & \\
\hline
\end{tabular}

FPG values was closely associated with long-term T2D remission after MS (Table 5).

3.4. Optimal Cutoff Values for C-Peptide AUC. Through the analysis of remission predictors, we noticed that C-peptide AUC has independent prognostic significance in T2D remission after MS, both in the short term and in the long term. To further explore the predictive ability of C-peptide AUC in T2D remission, we drew ROC curves (Figure 1). The optimal cutoff values for C-peptide AUC in predicting T2D remission were $30.93 \mathrm{ng} / \mathrm{ml}$, with areas under the ROC curves of 0.674 and 0.623 in the short term and long term, respectively (Table 6).

3.5. ACF Scoring System. Integrating our results above and the data published by other researchers, we hypothesized that a multidimensional grading system that assessed age, FPG, and C-peptide AUC would better categorize the illness 
TABLE 4: Univariate analysis of prolonged remission predictors.

\begin{tabular}{|c|c|c|c|}
\hline & Remission group $(N=19)$ & Nonremission group $(N=31)$ & $P$ value \\
\hline Age (yr) & & & 0.274 \\
\hline$<40$ & $4(66.67 \%)$ & $2(33.33 \%)$ & \\
\hline$\geq 40$ & $15(34.09 \%)$ & $29(65.91 \%)$ & \\
\hline Gender & & & 0.085 \\
\hline Female & $4(22.22 \%)$ & $14(77.78 \%)$ & \\
\hline Male & $15(47.87 \%)$ & $17(53.13 \%)$ & \\
\hline Duration (yr) & & & 0.121 \\
\hline$\leq 5$ & $11(50.00 \%)$ & $11(50.00 \%)$ & \\
\hline$>5$ & $8(28.57 \%)$ & $20(71.43 \%)$ & \\
\hline Surgical procedures & & & 0.759 \\
\hline Sleeve gastrectomy & $2(66.67 \%)$ & $1(33.33 \%)$ & \\
\hline Gastric bypass & $20(40.00 \%)$ & $30(60.00 \%)$ & \\
\hline BMI $\left(\mathrm{kg} / \mathrm{m}^{2}\right)$ & & & 0.057 \\
\hline$<27.5$ & $6(28.57 \%)$ & $15(71.43 \%)$ & \\
\hline $27.5-32.5$ & $8(34.78 \%)$ & $15(65.22 \%)$ & \\
\hline$\geq 32.5$ & $5(83.33 \%)$ & $1(16.67 \%)$ & \\
\hline $\mathrm{TG}(\mathrm{mmol} / \mathrm{L})$ & & & 0.923 \\
\hline$\geq 1.7$ & $12(37.50 \%)$ & $20(62.50 \%)$ & \\
\hline$<1.7$ & $7(38.89 \%)$ & $11(61.11 \%)$ & \\
\hline $\mathrm{CHOL}(\mathrm{mmol} / \mathrm{L})$ & & & 0.280 \\
\hline$\geq 4.14$ & $15(42.86 \%)$ & $20(57.14 \%)$ & \\
\hline$<4.14$ & $4(26.67 \%)$ & $11(73.33 \%)$ & \\
\hline $\mathrm{LDL}(\mathrm{mmol} / \mathrm{L})$ & & & 0.564 \\
\hline$\geq 2.59$ & $8(33.33 \%)$ & $16(66.67 \%)$ & \\
\hline$<2.59$ & $11(42.31 \%)$ & $15(57.69 \%)$ & \\
\hline $\mathrm{HDL}(\mathrm{mmol} / \mathrm{L})$ & & & 0.115 \\
\hline$>1.29$ & $6(66.67 \%)$ & $3(33.33 \%)$ & \\
\hline$\leq 1.29$ & $13(31.71 \%)$ & $28(68.29 \%)$ & \\
\hline FPG $(\mathrm{mmol} / \mathrm{L})$ & & & $0.043^{*}$ \\
\hline$<7.0$ & $11(55.00 \%)$ & $9(45.00 \%)$ & \\
\hline$\geq 7.0$ & $8(26.67 \%)$ & $22(73.33 \%)$ & \\
\hline HbAlc (\%) & & & $0.049^{*}$ \\
\hline$<6.5$ & $2(28.57 \%)$ & $5(71.43 \%)$ & \\
\hline $6.5 \leq \mathrm{HbA} 1 \mathrm{c}<9$ & $15(51.72 \%)$ & $14(48.28 \%)$ & \\
\hline$\geq 9$ & $2(14.29 \%)$ & $12(85.71 \%)$ & \\
\hline FCP (ng/mL) & $(N=19)$ & $(N=31)$ & 0.923 \\
\hline$\leq 2.63$ & $12(37.00 \%)$ & $20(62.50 \%)$ & \\
\hline$>2.63$ & $7(38.89 \%)$ & $11(61.11 \%)$ & \\
\hline$\Delta \mathrm{C} 30(\mathrm{ng} / \mathrm{mL})$ & $(N=18)$ & $(N=30)$ & 0.823 \\
\hline$<1.01$ & $9(36.00 \%)$ & $16(64.00 \%)$ & \\
\hline$\geq 1.01$ & $9(39.13 \%)$ & $14(60.87 \%)$ & \\
\hline$\Delta \mathrm{C} 120(\mathrm{ng} / \mathrm{mL})$ & $(N=19)$ & $(N=31)$ & 0.777 \\
\hline$\leq 3.08$ & $13(39.39 \%)$ & $20(60.61 \%)$ & \\
\hline$>3.08$ & $6(35.29 \%)$ & $11(64.71 \%)$ & \\
\hline$\Delta \mathrm{C} 30 / \Delta \mathrm{G} 30(\mu \mathrm{g} / \mathrm{mmol})$ & $(N=18)$ & $(N=30)$ & 0.654 \\
\hline$\leq 0.27$ & $9(34.62 \%)$ & $17(65.38 \%)$ & \\
\hline$>0.27$ & $9(40.91 \%)$ & $13(59.09 \%)$ & \\
\hline$\Delta \mathrm{C} 120 / \Delta \mathrm{G} 120(\mu \mathrm{g} / \mathrm{mmol})$ & $(N=19)$ & $(N=31)$ & 0.812 \\
\hline$\leq 0.42$ & $11(36.67 \%)$ & $19(63.33 \%)$ & \\
\hline$>0.42$ & $8(40.00 \%)$ & $12(60.00 \%)$ & \\
\hline HOMA- $\beta(\mathrm{CP}, \mathrm{DM})(\%)$ & $(N=19)$ & $(N=31)$ & 0.233 \\
\hline$\leq 52.71$ & $9(31.03 \%)$ & $20(68.97 \%)$ & \\
\hline$>52.71$ & $10(47.62 \%)$ & $11(52.38 \%)$ & \\
\hline HOMA-IR (CP) & $(N=19)$ & $(N=31)$ & 0.640 \\
\hline$\leq 2.08$ & $11(35.48 \%)$ & $20(64.52 \%)$ & \\
\hline$>2.08$ & $8(42.11 \%)$ & $11(57.89 \%)$ & \\
\hline C-peptide AUC (ng/ml) & $(N=18)$ & $(N=30)$ & $0.045^{*}$ \\
\hline$\leq 30.93$ & $8(26.67 \%)$ & $22(73.33 \%)$ & \\
\hline$>30.93$ & $10(55.56 \%)$ & $8(44.44 \%)$ & \\
\hline $\operatorname{UmAlb}(\mathrm{mg} / \mathrm{L})$ & $(N=16)$ & $(N=28)$ & 0.596 \\
\hline
\end{tabular}


TABle 4: Continued.

\begin{tabular}{lcc}
\hline & Remission group $(N=19)$ & Nonremission group $(N=31)$ \\
\hline$<20$ & $6(46.15 \%)$ & $7(53.85 \%)$ \\
$\geq 20$ & $10(32.66 \%)$ & $21(67.74 \%)$ \\
Preoperative insulin use & $10(33.33 \%)$ & $20(66.67 \%)$ \\
Yes & $9(45.00 \%)$ & $11(55.00 \%)$ \\
No & & 0.405 \\
\hline
\end{tabular}

${ }^{*} P<0.05$; indexes of islet function (FCP, $\Delta \mathrm{C} 30, \Delta \mathrm{C} 120, \Delta \mathrm{C} 30 / \Delta \mathrm{G} 30, \Delta \mathrm{C} 120 / \Delta \mathrm{G} 120$, HOMA- $\beta$ (CP, DM), HOMA-IR (CP), and C-peptide AUC) were grouped by median.

TABLE 5: Binary logistic regression of prolonged remission predictors.

\begin{tabular}{|c|c|c|c|}
\hline & $P$ value & OR & $\mathrm{CI}$ \\
\hline $\begin{array}{l}\text { C-peptide AUC (ng/ml) } \\
\quad \leq 30.93 \\
>30.93\end{array}$ & $\begin{array}{c}0.002 \\
\text { Reference }\end{array}$ & 8.139 & $1.962-33.762$ \\
\hline $\begin{array}{l}\text { FPG }(\mathrm{mmol} / \mathrm{L}) \\
\quad \geq 7.0 \\
\quad<7.0\end{array}$ & $\begin{array}{c}0.026^{*} \\
\text { Reference }\end{array}$ & 4.517 & $1.113-18.333$ \\
\hline $\begin{array}{l}\text { Gender } \\
\quad \text { Female } \\
\text { Male }\end{array}$ & $\begin{array}{c}0.078 \\
\text { Reference }\end{array}$ & & \\
\hline $\begin{array}{l}\mathrm{HbA} 1 \mathrm{c}(\%) \\
\quad<6.5 \\
\quad 6.5 \leq \mathrm{HbA} 1 \mathrm{c}<9 \\
\geq 9\end{array}$ & $\begin{array}{c}0.976 \\
\text { Reference }\end{array}$ & & \\
\hline $\begin{array}{l}\text { BMI }\left(\mathrm{kg} / \mathrm{m}^{2}\right) \\
\quad<27.5 \\
27.5-32.5 \\
\geq 32.5\end{array}$ & $\begin{array}{c}0.606 \\
\text { Reference }\end{array}$ & & \\
\hline
\end{tabular}

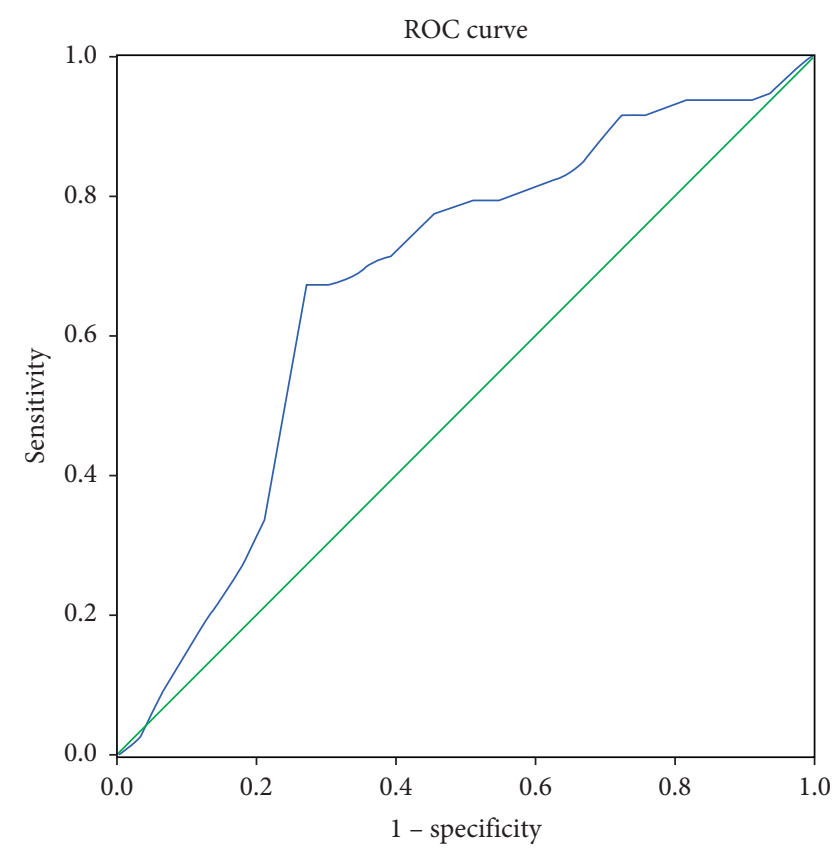

(a)

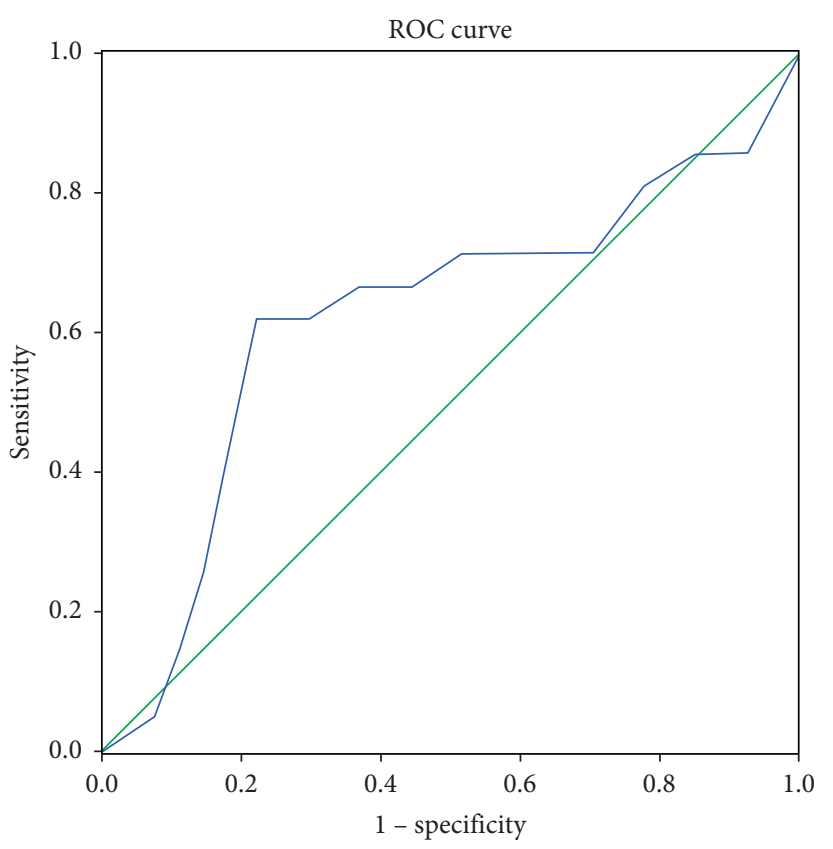

(b)

FIgURE 1: ROC curve of C-peptide AUC in predicting T2D remission. (a) The area under the ROC curve for prediction of short-term T2D remission using C-peptide AUC was 0.674 (specificity: 67.3\%; sensitivity: 75.8\%). (b) The area under the ROC curve for prediction of longterm T2D remission using C-peptide AUC was 0.623 (specificity: $61.9 \%$; sensitivity: $81.5 \%$ ). 
TABLE 6: ROC analysis of C-peptide AUC in remission prediction.

\begin{tabular}{lccrrr}
\hline & Optimal cutoff value $(\mathrm{ng} / \mathrm{ml})$ & Sensitivity $(\%)$ & Specificity (\%) & Youden's index & AUC \\
\hline Short-term remission & 30.93 & 67.3 & 75.8 & 0.431 & 0.674 \\
Prolonged remission & 30.93 & 61.9 & 81.5 & 0.434 & 0.623 \\
\hline
\end{tabular}

AUC: area under the ROC curve.

and predict the success of MS. The scoring system was abbreviated as ACF according to the initials of the indexes (Table 7). Applying the ACF score in this cohort (patients lacking C-peptide AUC values were excluded), we found that patients with a greater ACF score had a higher remission rate than other patients (Table 8). Patients with ACF scores ranging from 6 to 9 had short-term remission rates of $100.00 \%$ and prolonged remission rates of $80.00 \%$, and those with scores ranging from 2 to 5 had remission rates of $61.11 \%$ and $50.00 \%$ in the short and long term, respectively, while patients who scored 0 had the lowest remission rate (28.00\% in the short term and $10.53 \%$ in the long term).

To verify the practical ability of the ACF scoring system, we also applied other scoring systems to this cohort. The distribution of ABCD, DiaRem, and IMS scores of the studied patients is shown in Table 8. We found that the patients were more uniformly distributed using the ACF score than using the IMS score. Although the ABCD and DiaRem scores distributed patients better than the IMS score, the difference in remission rate among groups was not significant like the ACF score.

3.6. ROC Curve Analysis. On applying the ROC analysis (Figure 2), we could see that the ACF score had the maximum AUC in both the short-term and the long-term T2D remission prediction. The differences between the ACF and IMS score in the short-term prediction and the difference between ACF and DiaRem or IMS scores in the long-term prediction were significant (Table 9).

\section{Discussion}

Metabolic surgery has been now widely accepted as a method of treatment for diabetes that is superior to medical intervention for weight loss and diabetes remission [37, 38]. Recently, Chinese scholars reported T2D remission rates after MS of approximately $63.6-73.2 \%[20,39,40]$. The remission rate of T2D after MS varied widely because of different follow-up lengths, participant features, severity of T2D, and so on. In this study, $60.92 \%$ (53 of 87) of patients experienced T2D resolution in 1-2 years following MS, and five of them had T2D recurrence thereafter. Of the $69.44 \%$ (50 of 72) of patients completing 4-6 years of follow-up, $38.00 \%$ (19 of 50) remained in prolonged remission.

It is worth noting that patients with $\mathrm{T} 2 \mathrm{D}$ are not all in the same situation, and the optimal candidates for MS should be identified. Although predictive factors for T2D remission after MS in Asian populations have already been published by other researchers, including young age, high BMI, short duration, lower levels of FPG and HbA1c, higher C-peptide concentration, and preoperative insulin use $[18,41-43]$, values of $\beta$-cell function, especially comprehensive indexes
TABLE 7: ACF score.

\begin{tabular}{lc}
\hline Factor & Score \\
\hline Age $(\mathrm{yr})$ & \\
$\quad \geq 40$ & 0 \\
$\quad<40$ & 4 \\
C-peptide AUC $(\mathrm{ng} / \mathrm{ml})$ & \\
$\quad \leq 30.93$ & 0 \\
$\quad>30.93$ & 3 \\
FPG $(\mathrm{mmol} / \mathrm{L})$ & \\
$\quad \geq 7.0$ & 0 \\
$\quad<7.0$ & 2 \\
Total & $0-9$ \\
\hline
\end{tabular}

reflecting $\beta$-cell function in T2D remission, were lacking. Progressive pancreatic $\beta$-cell failure is a natural course of diabetes and East Asian patients have been proved to have a worse $\beta$-cell function at the beginning of T2D. Focusing on the value of residual $\beta$-cell function, we explored the remission predictors of T2D remission after MS in mainland Chinese patients.

In what concerns $\beta$-cell function indexes, univariate regression showed that patients with higher FCP, higher $\Delta \mathrm{C} 120$, higher HOMA- $\beta$ (CP, DM), higher HOMA-IR (CP), and higher C-peptide AUC levels (grouped by median) have a much higher short-term remission rate than those in the lower groups, which indicates that $\beta$-cell function indexes play important roles in predicting short-term $\mathrm{T} 2 \mathrm{D}$ remission after MS. Among them, the difference in C-peptide AUC is the most significant $(P<0.001)$. In the long-term follow-up, only patients with higher C-peptide AUC have a significant higher remission rate than those in the lower group, while there was no significant difference in the remission rate among groups classified by other $\beta$-cell function indexes. Patients with C-peptide AUC $>30.93 \mathrm{ng} / \mathrm{ml}$ have a 2 -fold possibility of T2D remission both in the short term $(80.93 \%$ vs. $39.02 \%)$ and in the long term $(55.56 \%$ vs. $26.67 \%)$. Moreover, as it was the only factor that showed significance in both the short- and long-term binary regression, we had to acknowledge the independent prognostic significance. Furthermore, we explored the optimal cutoff point for C-peptide AUC in predicting T2D remission. ROC curves showed that the optimal cutoff value of C-peptide AUC in prediction was $30.93 \mathrm{ng} / \mathrm{ml}$, with a specificity of $67.3 \%$ and sensitivity of $75.8 \%$ in the short-term T2D remission and with a specificity of $61.9 \%$ and sensitivity of $81.5 \%$ in the long-term T2D remission, respectively. The area under the ROC curves ( 0.674 and 0.623 for the short- and long-term, respectively) had a moderate ability to predict remission. It has been demonstrated that C-peptide AUC has an association with higher T2D remission rate in Western patients whose average BMIs were higher than $44 \mathrm{~kg} / \mathrm{m}^{2}$ in the short- 
TABle 8: Predictive ability of scores.

\begin{tabular}{|c|c|c|c|c|c|c|c|}
\hline \multirow{2}{*}{ Model } & \multirow{2}{*}{ Total points } & \multicolumn{3}{|c|}{ Short-term data $(N=82)$} & \multicolumn{3}{|c|}{ Long-term data $(N=48)$} \\
\hline & & Remission $(N)$ & Nonremission $(N)$ & Remission rate (\%) & Remission $(N)$ & Nonremission $(N)$ & Remission rate (\%) \\
\hline \multirow{3}{*}{$\mathrm{ACF}$} & 0 & 7 & 18 & 28.00 & 2 & 17 & 10.53 \\
\hline & $2-5$ & 22 & 14 & 61.11 & 12 & 12 & 50.00 \\
\hline & $6-9$ & 20 & 0 & 100.00 & 4 & 1 & 80.00 \\
\hline \multirow{5}{*}{$\mathrm{ABCD}$} & $0-2$ & 11 & 23 & 32.35 & 6 & 20 & 23.08 \\
\hline & $3-4$ & 17 & 7 & 70.83 & 6 & 7 & 46.15 \\
\hline & $5-6$ & 12 & 1 & 92.31 & 5 & 1 & 83.33 \\
\hline & $7-8$ & 8 & 2 & 80.00 & 1 & 2 & 33.33 \\
\hline & $9-10$ & 1 & 0 & 100.00 & - & - & - \\
\hline \multirow{5}{*}{ DiaRem } & $0-2$ & 7 & 11 & 38.89 & 3 & 7 & 30.00 \\
\hline & $3-7$ & 13 & 14 & 48.15 & 7 & 12 & 36.84 \\
\hline & $8-12$ & 7 & 5 & 58.33 & 3 & 6 & 33.33 \\
\hline & $13-17$ & 16 & 2 & 88.89 & 5 & 3 & 62.50 \\
\hline & $18-22$ & 6 & 11 & 85.71 & 0 & 2 & 0.00 \\
\hline \multirow{3}{*}{ IMS } & 25 & 2 & 8 & 20.00 & 1 & 4 & 20.00 \\
\hline & $25-95$ & 42 & 24 & 63.64 & 16 & 25 & 39.02 \\
\hline & $\geq 95$ & 5 & 1 & 83.33 & 1 & 1 & 50.00 \\
\hline
\end{tabular}

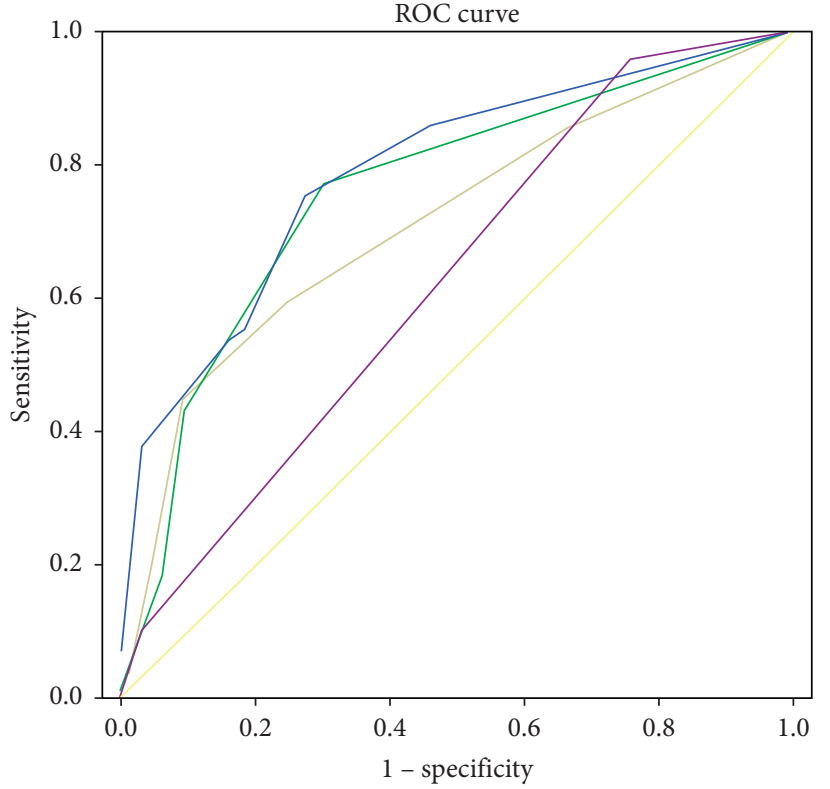

Source of the curve

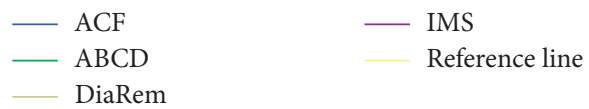

(a)

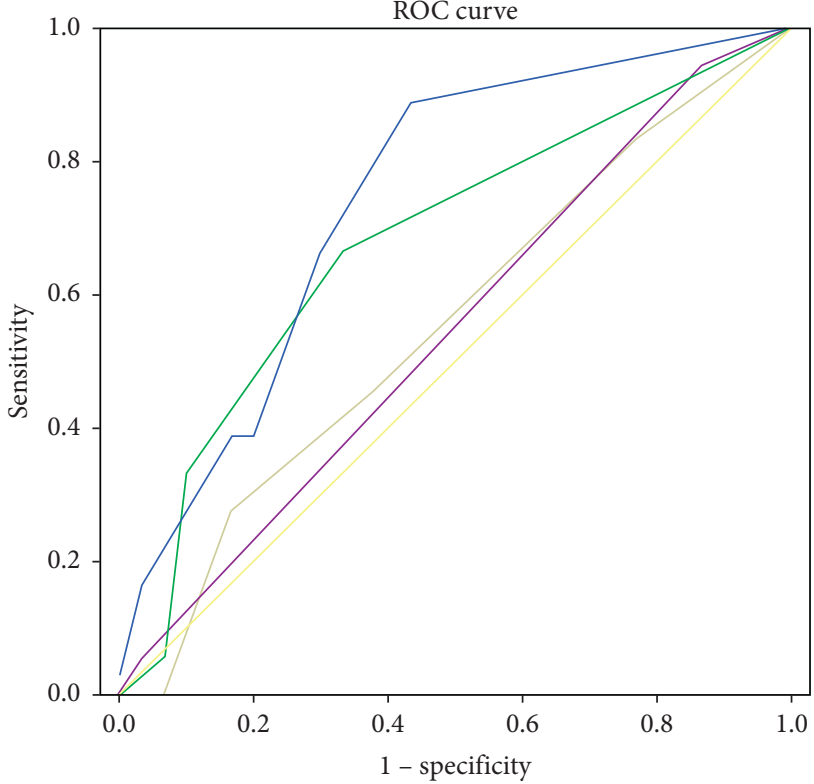

Source of the curve

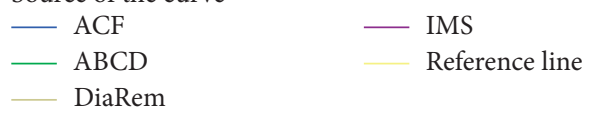

(b)

FIGURE 2: ROC curve analysis of ACF, ABCD, Diarem, and IMS scores. (a) In the short-term T2D remission prediction, the area under the ROC curves using the ACF score (the blue line) is $0.793, P<0.001^{*}$; the area under the ROC curves using the ABCD score (the green line) is $0.762, P<0.001^{*}$; the area under the ROC curves using the DiaRem score (the brown line) is $0.715, P=0.001^{*}$; and the area under the ROC curves using the IMS score (the purple line) is $0.625, P=0.056$. (b) In the long-term T2D remission prediction, the area under the ROC curves using the ACF score (the blue line) is $0.748, P=0.004^{*}$; the area under the ROC curves using the ABCD score (the green line) is 0.681 , $P=0.038^{*}$; the area under the ROC curves using the DiaRem score (the brown line) is $0.556, P=0.086$; and the area under the ROC curves using the IMS score (the purple line) is $0.547, P=0.086$.

term follow-up [23, 34]. In the present study, we identified that C-peptide AUC is an independent predictor of T2D remission after MS in Chinese patients both in the short term and in the long term.
Some studies verified that age plays a role in $\mathrm{T} 2 \mathrm{D}$ remission prediction after MS $[28,41]$. In the present study, binary logistic regression showed that age younger than 40 years facilitates short-term T2D remission, which confirms 
TABLE 9: Comparison of ROC analysis of different models.

\begin{tabular}{ccccc}
\hline & Model & AUC & $P$ value & $95 \%$ CI \\
\hline & ACF & 0.793 & $<0.001^{*}$ & $0.696-0.890$ \\
Short-term data & ABCD & 0.762 & $<0.001^{*}$ & $0.655-0.869$ \\
& DiaRem & 0.715 & $0.001^{*}$ & $0.603-0.826$ \\
& IMS & $0.625^{\S}$ & 0.056 & $0.500-0.750$ \\
\hline & ACF & 0.748 & $0.004^{*}$ & $0.607-0.889$ \\
Long-term data & ABCD & 0.681 & $0.038^{*}$ & $0.387-0.724$ \\
& DiaRem & $0.556^{\S}$ & 0.086 & $0.380-0.715$ \\
& IMS & $0.547^{\S}$ & 0.086 & $0.520-0.841$ \\
\hline
\end{tabular}

AUC: area under the ROC curve. ${ }^{\$}$ Compared to ACF, $P$ value of AUC $<0.05$. ${ }^{*} P<0.05$.

that it is an independent predictor of short-term T2D remission after MS. In the long-term follow-up, younger patients (age $<40$ years) had a higher remission rate than the older patients $(P=0.274)$.

Regarding FPG, there were inconsistencies among studies. The level of fasting glucose value was found to be associated with T2D remission in different reports $[20,34,44]$. The present study showed that patients with elevated level of FPG values had lower remission rates both in the short term and long term, and the significance remained in the long-term binary regression.

The other parameters that were significant in the univariate regression, including $\mathrm{BMI}$, surgical procedures, the duration of T2D, HbA1c, and presurgical insulin usage, have all been reported by other studies [44-47]. In our study, the remission rate of patients with higher BMI was significantly higher in 1-2 years after surgery. However, the difference was not significant in the binary regression nor in the long-term remission prediction. Studies have shown that Asian patients with BMIs below $35 \mathrm{~kg} / \mathrm{m}^{2}$ obtain successful glycemic control after bariatric surgery $[19,48]$. Therefore, we speculated that BMI may not be the most important factor in T2D remission prediction. Besides, some studies have shown that weight loss after MS may be a predictive factor for T2D remission in addition to preoperative BMI $[18,49]$. In the present study, we mainly discussed the predictive value of preoperation factors, so we did not include the EWL\% or TWL\%. In this cohort, over 95\% of the patients who chose GB did so before 2014, while approximately $70 \%$ of patients underwent SG thereafter. Therefore, different enrolment standards and advances in surgical techniques and postsurgery management may have contributed to the different remission rates between the two groups. In addition, the preoperative data showed a significant difference between the two groups, and only a minority of patients underwent SG (28.7\%). Therefore, we did not include surgical procedures in the binary logistic regression to minimize the deviation. A shorter duration of T2D was shown to be significantly associated with a higher remission rate in the short-term follow-up. And, we verified that longer T2D duration was correlated with worse $\beta$-cell function (FCP, $\Delta \mathrm{C} 30 / \Delta \mathrm{G} 30$, and C-peptide AUC) and lower insulin resistance (HOMA-IR (CP)). And, presurgery insulin use was found to be associated with only short-term remission. Longer T2D duration and presurgery insulin use both partly imply worse $\beta$-cell function and thus may be corrected by other indexes in the binary logistic analysis. Additionally, $\mathrm{HbAlc}$ failed to show significance in the binary regression. No association between T2D remission and sex, lipid biomarkers, or indicators reflecting microvascular diseases (UmAlb) was found herein.

To select the patients best suited to MS, identifying preoperative predictors of $\mathrm{T} 2 \mathrm{D}$ remission can lead to improved outcomes, and researchers have proposed several prediction models, such as the ABCD, DiaRem, and IMS scoring systems. Interestingly, $\beta$-cell function was not included in the scores except those for the ABCD. According to our study, $\beta$-cell function, especially C-peptide AUC, is a decisive predictive factor. Therefore, we tried to establish our own scoring system with regard to the regression analysis. We included three indexes in the scoring system according to regression analysis: age, FPG, and C-peptide AUC. Patients with a greater ACF score had a higher remission rate in both the short term and long term. We classified patients into three groups according to the ACF score. Those with scores ranging from 6-9 had the highest remission rate $(100.00 \%$ and $80.00 \%$ in the short term and long term, respectively), and patients in the second group, whose score ranged from 2-5, achieved a remission rate of $61.11 \%$ in the short term and $50.00 \%$ in the long term. The patients with the lowest scores had the lowest remission rates $28.00 \%$ in the short term and $10.53 \%$ in the long term). Then, we compared our scoring system with others to verify the prognostic accuracy. The ACF score showed more significance in predicting the remission rate among the groups compared with the $\mathrm{ABCD}$ and DiaRem scores and a more uniform distribution compared with the IMS score. According to the ROC curves, the ACF score had the maximum AUC both in the short- and long-term remission prediction. The reason why the IMS and DiaRem scores did not show excellent predictive abilities in this cohort may be the different race of the patients and significant differences in baseline characteristics. Both of the original and validated data in those two studies came from severely obese Caucasians, with BMIs higher than $45 \mathrm{~kg} / \mathrm{m}^{2}$, while our study was based on Chinese participants with an average BMI of $31.29 \pm 6.51 \mathrm{~kg} / \mathrm{m}^{2}$. We noticed that when compared with the ABCD score, our study showed superiority in grading of remission rates among groups and a larger AUC. The ABCD scoring system included FCP as an index of $\beta$-cell function, and our study showed that C-peptide AUC is a superior predictor than FCP in T2D remission. Another difference between the ACF and ABCD scores was that the latter included T2D duration, which was not considered as an independent predictive factor in this cohort.

This is the first prediction model of T2D remission after MS based on a mainland Chinese population, and it notably includes C-peptide AUC, an index of residual $\beta$-cell function, for choosing the optimal metabolic procedure among the two common surgical procedures. The ACF scoring system showed excellent predictive value in the present study. Undoubtedly, it still needs to be validated externally to verify its feasibility. 
The limitations of this study include being a single-center study and having a relatively small number of study subjects. These factors precluded us from a more complicated statistical analysis and might also have weakened the statistical power to a certain degree. However, the ACF score is simple to calculate, which makes it a practical tool and with potentially widespread applicability.

\section{Conclusions}

In summary, the present study has shown that presurgery age, C-peptide AUC, and FPG are independent predictors of T2D remission after MS. Among these, C-peptide AUC plays a decisive role in both short- and long-term remission prediction, and the optimal cutoff value for C-peptide AUC in predicting T2D remission was $30.93 \mathrm{ng} / \mathrm{ml}$, with moderate predictive values. The ACF score is a simple reliable system that can predict $\mathrm{T} 2 \mathrm{D}$ remission among Chinese patients.

\section{Data Availability}

The data used to support the findings of this study are available from the corresponding author upon request.

\section{Conflicts of Interest}

The authors declare that there are no conflicts of interest.

\section{Authors' Contributions}

Study concepts were prepared by Zhaohui Mo. The study was designed by Zhaohui Mo and Yufang Luo. Data acquisition was performed by Yufang Luo, Honghui He, and Shaoli Zhao. Quality control of data and algorithms was carried out by Honghui $\mathrm{He}$ and Youbo Yang. Data analysis and interpretation was conducted by Yufang Luo and Zhaohui Mo. Statistical analysis was performed by Yufang Luo and $\mathrm{Zi}$ Guo. The manuscript was prepared and edited by Yufang Luo and Zhaohui Mo. All the authors approved the final version of the manuscript.

\section{Acknowledgments}

This work was supported by the National Natural Science Foundation of China (No. 81670769) and Talent Fund Project of Third Xiangya Hospital (2014-2018).

\section{References}

[1] L. Wang, P. Gao, M. Zhang et al., "Prevalence and ethnic pattern of diabetes and prediabetes in China in 2013," JAMA, vol. 317, no. 24, pp. 2515-2523, 2017.

[2] J. C. Chan, V. Malik, W. Jia et al., "Diabetes in Asia: epidemiology, risk factors, and pathophysiology," JAMA, vol. 301, no. 20, pp. 2129-2140, 2009.

[3] G. Mingrone, S. Panunzi, A. De Gaetano et al., "Bariatric surgery versus conventional medical therapy for type 2 diabetes," The New England Journal of Medicine, vol. 366, no. 17, pp. 1577-1585, 2012.
[4] S. Ikramuddin, J. Korner, W. J. Lee et al., "Roux-en-Y gastric bypass vs intensive medical management for the control of type 2 diabetes, hypertension, and hyperlipidemia: the diabetes surgery study randomized clinical trial," Jama, vol. 309, no. 21, pp. 2240-2249, 2013.

[5] P. R. Schauer, S. R. Kashyap, K. Wolski et al., "Bariatric surgery versus intensive medical therapy in obese patients with diabetes," The New England Journal of Medicine, vol. 366, no. 17, pp. 1567-1576, 2012.

[6] D. E. Cummings, D. E. Arterburn, E. O. Westbrook et al., "Gastric bypass surgery vs intensive lifestyle and medical intervention for type 2 diabetes: the CROSSROADS randomised controlled trial," Diabetologia, vol. 59, no. 5, pp. 945-953, 2016.

[7] S. R. Kashyap, D. L. Bhatt, K. Wolski et al., "Metabolic effects of bariatric surgery in patients with moderate obesity and type 2 diabetes: analysis of a randomized control trial comparing surgery with intensive medical treatment," Diabetes Care, vol. 36, no. 8, pp. 2175-2182, 2013.

[8] A. P. Courcoulas, S. H. Belle, R. H. Neiberg et al., "Three-year outcomes of bariatric surgery vs lifestyle intervention for type 2 diabetes mellitus treatment: a randomized clinical trial," JAMA Surgery, vol. 150, no. 10, pp. 931-940, 2015.

[9] Z. Khorgami, S. Shoar, A. A. Saber, C. A. Howard, G. Danaei, and G. M. Sclabas, "Outcomes of bariatric surgery versus medical management for type 2 diabetes mellitus: a metaanalysis of randomized controlled trials," Obesity Surgery, vol. 29, no. 3, pp. 964-974, 2019.

[10] G. Mingrone, G. Nolfe, G. Castagneto Gissey et al., "Circadian rhythms of GIP and GLP1 in glucose-tolerant and in type 2 diabetic patients after biliopancreatic diversion," Diabetologia, vol. 52, no. 5, 2009.

[11] C. Guida, S. Stephen, R. Guitton, and R. D. Ramracheya, "The role of PYY in pancreatic islet physiology and surgical control of diabetes," Trends in Endocrinology and Metabolism: TEM, vol. 28, no. 8, pp. 626-636, 2017.

[12] S. S. Evers, D. A. Sandoval, and R. J. Seeley, "The physiology and molecular underpinnings of the effects of bariatric surgery on obesity and diabetes," Annual Review of Physiology, vol. 79, pp. 313-334, 2017.

[13] B. Pérez-Pevida, J. Escalada, A. D. Miras, and G. Frühbeck, "Mechanisms underlying type 2 diabetes remission after metabolic surgery," Front Endocrinol (Lausanne), vol. 10, 2019.

[14] Z. Khorgami, S. Shoar, A. Andalib, A. Aminian, S. A. Brethauer, and P. R. Schauer, "Trends in utilization of bariatric surgery, 2010-2014: sleeve gastrectomy dominates," Surgery for Obesity and Related Diseases, vol. 13, no. 5, pp. 774-778, 2017.

[15] N. Hamza, M. H. Abbas, A. Darwish, Z. Shafeek, J. New, and B. J. Ammori, "Predictors of remission of type 2 diabetes mellitus after laparoscopic gastric banding and bypass," Surgery for Obesity and Related Diseases, vol. 7, no. 6, pp. 691-696, 2011.

[16] T. C. Hall, M. G. Pellen, P. C. Sedman, and P. K. Jain, "Preoperative factors predicting remission of type 2 diabetes mellitus after Roux-en-Y gastric bypass surgery for obesity," Obesity Surgery, vol. 20, no. 9, pp. 1245-1250, 2010.

[17] M. T. Hayes, L. A. Hunt, J. Foo, Y. Tychinskaya, and R. S. Stubbs, "A model for predicting the resolution of type 2 diabetes in severely obese subjects following Roux-en Y gastric bypass surgery," Obesity Surgery, vol. 21, no. 7, pp. 910-916, 2011. 
[18] J. Y. Park and Y. J. Kim, "Prediction of diabetes remission in morbidly obese patients after roux-en-Y gastric bypass," Obesity Surgery, vol. 26, no. 4, pp. 749-756, 2016.

[19] Y. Seki, K. Kasama, K. Yasuda, R. Yokoyama, J. P. Porciuncula, and Y. Kurokawa, "The effects of laparoscopic sleeve gastrectomy with duodenojejunal bypass on Japanese patients with $\mathrm{BMI}<35 \mathrm{~kg} / \mathrm{m}^{2}$ on type 2 diabetes mellitus and the prediction of successful glycemic control," Obesity Surgery, vol. 28, no. 8, pp. 2429-2438, 2018.

[20] H. Yu, J. Di, Y. Bao et al., "“Visceral fat area as a new predictor of short-term diabetes remission after Roux-en-Y gastric bypass surgery in Chinese patients with a body mass index less than $35 \mathrm{~kg} / \mathrm{m} 2$ " Surgery for obesity and related diseases," Official Journal of the American Society for Bariatric Surgery, vol. 11, no. 1, pp. 6-11, 2015.

[21] R. Blackstone, J. C. Bunt, M. C. Cortes, and H. J. Sugerman, "Type 2 diabetes after gastric bypass: remission in five models using HbA1c, fasting blood glucose, and medication status," Surgery for Obesity and Related Diseases, vol. 8, no. 5, pp. 548-555, 2012.

[22] W. J. Lee, K. Chong, K. H. Ser et al., "C-peptide predicts the remission of type 2 diabetes after bariatric surgery," Obesity Surgery, vol. 22, no. 2, pp. 293-298, 2012.

[23] A. Mallipedhi, T. Min, S. L. Prior et al., "Association between the preoperative fasting and postprandial C-peptide AUC with resolution of type 2 diabetes 6 months following bariatric surgery," Metabolism: Clinical and Experimental, vol. 64, no. 11, pp. 1556-1563, 2015.

[24] J. B. Dixon, L. M. Chuang, K. Chong et al., "Predicting the glycemic response to gastric bypass surgery in patients with type 2 diabetes," Diabetes Care, vol. 36, no. No. 1, pp. 20-26, 2013.

[25] A. M. Ramos-Levi, P. Matia, L. Cabrerizo et al., "Statistical models to predict type 2 diabetes remission after bariatric surgery," Journal of Diabetes, vol. 6, no. 5, pp. 472-477, 2014.

[26] W. J. Lee, K. Y. Hur, M. Lakadawala et al., "Predicting success of metabolic surgery: age, body mass index, C-peptide, and duration score," Surgery for Obesity and Related Diseases, vol. 9, no. 3, pp. 379-384, 2013.

[27] A. Aminian, S. A. Brethauer, A. Andalib et al., "Individualized metabolic surgery score: procedure selection based on diabetes severity," Annals of Surgery, vol. 266, no. 4, pp. 650-657, 2017.

[28] C. D. Still, G. C. Wood, P. Benotti et al., "Preoperative prediction of type 2 diabetes remission after Roux-en-Y gastric bypass surgery: a retrospective cohort study," Lancet Diabetes Endocrinol, vol. 2, no. 1, pp. 38-45, 2014.

[29] U. Surendra, N. Gupta, D. Modi Kirtikumar et al., "Prediction of remission after metabolic surgery using a novel scoring system in type 2 diabetes-a retrospective cohort study," Journal of Diabetes \& Metabolic Disorders, vol. 13, no. 1, 2014.

[30] Y. Xu, L. Wang, J. He et al., "Prevalence and control of diabetes in Chinese adults," JAMA, vol. 310, no. 9, pp. 948-959, 2013.

[31] J. B. Dixon, P. Zimmet, K. G. Alberti, and F. Rubino, "Bariatric surgery: an IDF statement for obese Type 2 diabetes," Surgery for Obesity and Related Diseases, vol. 7, no. 4, pp. 433-447, 2011.

[32] American Diabetes Association, "Obesity management for the treatment of type 2 diabetes: standards of medical care in diabetes-2019," Diabetes care, vol. 42, no. 1, pp. S81-S89, 2019.

[33] E. O. Aarts, J. Janssen, I. M. C. Janssen, F. J. Berends, D. Telting, and H. de Boer, "Preoperative fasting plasma C-peptide level may help to predict diabetes outcome after gastric bypass surgery," Obesity Surgery, vol. 23, no. 7, pp. 867-873, 2013.

[34] P. Souteiro, S. Belo, J. S. Neves et al., "Preoperative beta cell function is predictive of diabetes remission after bariatric surgery,” Obesity Surgery, vol. 27, no. 2, pp. 288-294, 2017.

[35] S. Cernea and M. Dobreanu, "Diabetes and beta cell function: from mechanisms to evaluation and clinical implications," Biochemia Medica, vol. 23, no. 3, pp. 266-280, 2013.

[36] X. Li, Z. G. Zhou, H. Y. Qi, X. Y. Chen, and G. Huang, "Replacement of insulin by fasting C-peptide in modified homeostasis model assessment to evaluate insulin resistance and islet beta cell function," Zhong nan da xue xue bao Yi xue ban = Journal of Central South University Medical Sciences, vol. 29, no. 4, pp. 419-423, 2004.

[37] D. E. Cummings and F. Rubino, "Metabolic surgery for the treatment of type 2 diabetes in obese individuals," Diabetologia, vol. 61, no. 2, pp. 257-264, 2018.

[38] A. H. Affinati, N. H. Esfandiari, E. A. Oral, and A. T. Kraftson, "Bariatric surgery in the treatment of type 2 diabetes," Current Diabetes Reports, vol. 19, no. 12, 2019.

[39] H. Zhang, X. Han, H. Yu, J. Di, P. Zhang, and W. Jia, "Effect of roux-en-Y gastric bypass on remission of T2D: medium-term follow-up in Chinese patients with different BMI obesity class," Obesity Surgery, vol. 27, no. 1, pp. 134-142, 2017.

[40] C. K. Huang, A. Shabbir, C. H. Lo, C. M. Tai, Y. S. Chen, and J. Y. Houng, "Laparoscopic Roux-en-Y gastric bypass for the treatment of type II diabetes mellitus in Chinese patients with body mass index of 25-35," Obesity Surgery, vol. 21, no. 9, pp. 1344-1349, 2011.

[41] M. H. Lee, W. J. Lee, K. Chong et al., "Predictors of long-term diabetes remission after metabolic surgery," Journal of Gastrointestinal Surgery: Official Journal of the Society for Surgery of the Alimentary Tract, vol. 19, no. 6, pp. 1015-1021, 2015.

[42] L. Zhao, W. Li, Z. Su, Y. Liu, L. Zhu, and S. Zhu, "Preoperative fasting C-peptide predicts type 2 diabetes mellitus remission in low-BMI Chinese patients after roux-en-Y gastric bypass," Journal of Gastrointestinal Surgery: Official Journal of the Society for Surgery of the Alimentary Tract, vol. 22, no. 10, pp. 1672-1678, 2018.

[43] V. L. P. de Oliveira, G. P. Martins, C. C. Mottin, J. Rizzolli, and R. Friedman, "Predictors of long-term remission and relapse of type 2 diabetes mellitus following gastric bypass in severely obese patients," Obesity Surgery, vol. 28, no. 1, pp. 195-203, 2018.

[44] S. Panunzi, L. Carlsson, A. De Gaetano et al., "Determinants of diabetes remission and glycemic control after bariatric surgery," Diabetes Care, vol. 39, no. 1, pp. 166-174, 2016.

[45] M. Robert, C. Ferrand-Gaillard, E. Disse et al., "Predictive factors of type 2 diabetes remission 1 year after bariatric surgery: impact of surgical techniques," Obesity Surgery, vol. 23, no. 6, pp. 770-775, 2013.

[46] P. C. Lee, K. W. Tham, S. Ganguly, H. C. Tan, A. K. H. Eng, and J. B. Dixon, "Ethnicity does not influence glycemic outcomes or diabetes remission after sleeve gastrectomy or gastric bypass in a multiethnic asian cohort," Obesity Surgery, vol. 28, no. 6, pp. 1511-1518, 2018.

[47] H. Yu, Y. Ni, Y. Bao et al., "Chenodeoxycholic acid as a potential prognostic marker for roux-en-Y gastric bypass in Chinese obese patients," The Journal of Clinical Endocrinology and Metabolism, vol. 100, no. 11, pp. 4222-4230, 2015.

[48] W. S. Rao, C. X. Shan, W. Zhang, D. Z. Jiang, and M. Qiu, “A meta-analysis of short-term outcomes of patients with type 2 diabetes mellitus and $\mathrm{BMI}</=35 \mathrm{~kg} / \mathrm{m}^{2}$ undergoing Roux-en- 
Y gastric bypass," World Journal of Surgery, vol. 39, no. 1, pp. 223-230, 2015.

[49] S. A. Brethauer, A. Aminian, H. Romero-Talamas et al., "Can diabetes be surgically cured? long-term metabolic effects of bariatric surgery in obese patients with type 2 diabetes mellitus," Annals of Surgery, vol. 258, no. 4, pp. 628-636, 2013. 